\title{
Light Dark Matter Searches with DarkMESA
}

M. Christmann ${ }^{a, b, \dagger, *}$, P. Achenbach ${ }^{a, b, c}$, S. Aulenbacher ${ }^{a}$, M. Biroth $^{a}$, S. Caiazza ${ }^{a}$, A. Denig ${ }^{a, b, c}$, L. Doria ${ }^{a, c}$, J. Geimer ${ }^{a}$, P. Gülker ${ }^{a}$, P. Klag ${ }^{a}$, M. Lauß ${ }^{a, b}$, M. Littich $^{a}$, S. Lunkenheimer ${ }^{a}$, T. Manoussos ${ }^{a}$, D. Markus ${ }^{a}$, M. Mauch $^{a, b}$, H. Merkel ${ }^{a, c}$, J. Müller $^{a}$, J. Schlaadt ${ }^{a}$, B. S. Schlimme ${ }^{a}$, C. Sfienti ${ }^{a, c}$, S. Stengel ${ }^{a, b}$ and C. Szyszka ${ }^{a}$ for the MAGIX collaboration

${ }^{a}$ Institute for Nuclear Physics, Johannes Gutenberg University Mainz, Johann-Joachim-Becher-Weg 45, 55128 Mainz, Germany

${ }^{b}$ Helmholtz Institute Mainz, GSI Helmholtzzentrum für Schwerionenforschung, Darmstadt, Johannes Gutenberg University Mainz, 55099 Mainz, Germany

${ }^{c}$ PRISMA ${ }^{+}$Cluster of Excellence, Johannes Gutenberg University Mainz, Staudingerweg 9, 55128 Mainz, Germany

E-mail: mircochr@students.uni-mainz.de

At the Institute for Nuclear Physics in Mainz the new electron accelerator MESA will go into operation within the next years. In the extracted beam operation $(150 \mathrm{MeV}, 150 \mu \mathrm{A})$ the P2 experiment will measure the weak mixing angle in electron-proton scattering. The high-power beam dump of this experiment and the 20,000 hours operation time are ideally suited for a parasitic dark sector experiment - DarkMESA.

The experiment is designed for the detection of Light Dark Matter (LDM) which in the simplest model couples to a massive vector particle, the dark photon $\gamma^{\prime}$. It can potentially be produced in the $\mathrm{P} 2$ beam dump by a process analogous to photon Bremsstrahlung and can then decay in Dark Matter (DM) particle pairs $\chi \bar{\chi}$ if kinematically allowed. A fraction of them scatter off electrons or nuclei in the DarkMESA calorimeter.

For the calorimeter, high-density $\mathrm{PbF}_{2}$ and lead glass SF5 Cherenkov radiators and photomultipliers from previous experiments will be used. In Phase A a prototype with $25 \mathrm{PbF}_{2}$ crystals and in Phase $\mathrm{B} \sim 2000 \mathrm{PbF}_{2}$ and SF5 crystals with a total active volume of $0.7 \mathrm{~m}^{3}$ are foreseen. For the prototype stage, a hermetic veto system with two layers of plastic scintillators and $1 \mathrm{~cm}$ of lead shielding is currently under development.

Within a MadGraph and Geant4 simulation the accessible parameter space was evaluated. The experimental setup was optimized and further concepts were investigated.

DarkMESA DRIFT is currently considered as an addition to the project. A Time Projection Chamber (TPC) filled with $\mathrm{CS}_{2}$ at low pressure will serve as DM detector. With the nuclear recoil threshold being in the $\mathrm{keV}$ range the accessible parameter space can be extended.

\footnotetext{
*** The European Physical Society Conference on High Energy Physics (EPS-HEP2021), *** *** 26-30 July $2021 * * *$

*** Online conference, jointly organized by Universität Hamburg and the research center DESY ***
}

\footnotetext{
*Speaker

${ }^{\dagger}$ Part of doctoral thesis.
} 


\section{Introduction}

The Institute for Nuclear Physics in Mainz houses the electron accelerator Mainz Microtron (MAMI) and is expanding its experimental possibilities with the new Mainz Energy recovering Superconducting Accelerator (MESA). The first beam is expected in 2023.

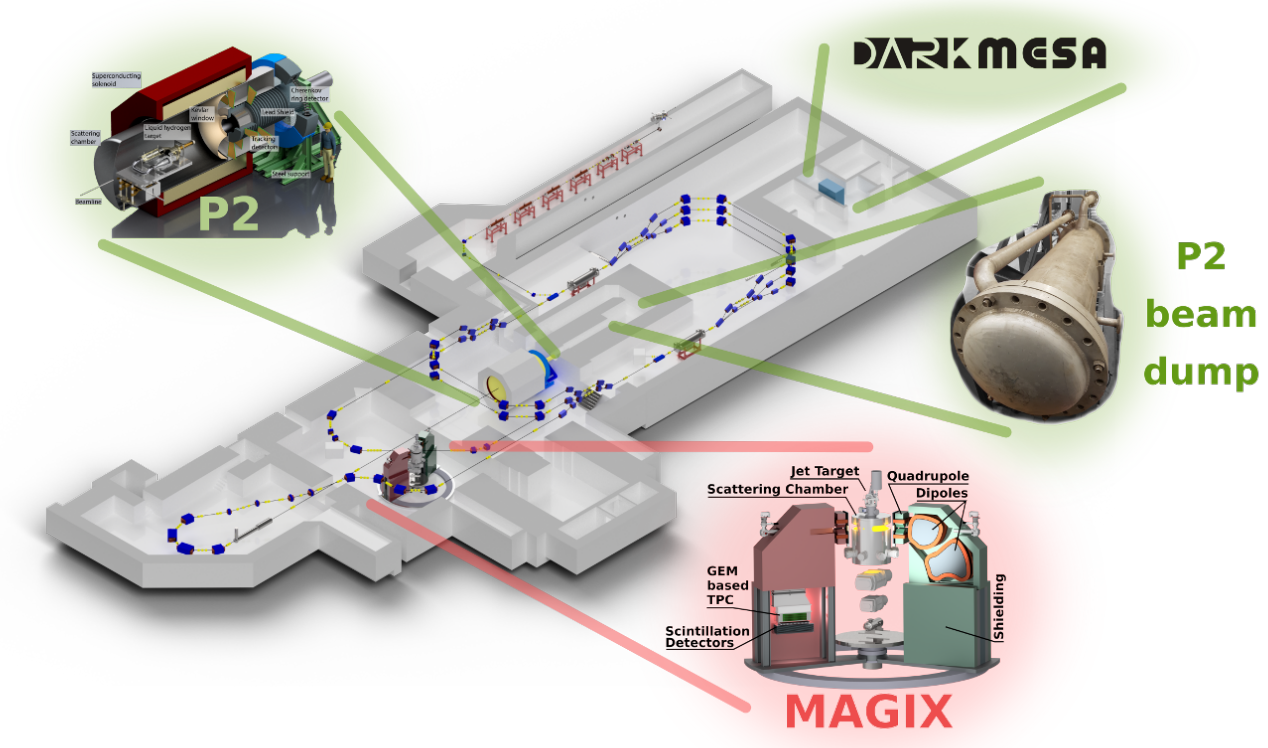

Figure 1: Model of the Mainz Energy recovering Superconducting Accelerator (MESA) [1] with enlarged views of the MAGIX experiment [2], the P2 experiment [3] and the P2 beam dump. (status 2021)

The accelerator will have two operating modes. An energy-recovering linac (ERL) mode with a beam energy up to $105 \mathrm{MeV}$ and a beam current of $1 \mathrm{~mA}$, and an extracted beam (EB) mode with a polarized beam of $150 \mathrm{MeV}$ and $150 \mu \mathrm{A}$. In each circulation the beam electrons gain up to $50 \mathrm{MeV}$ energy in two superconducting cryomodules.

After two recirculations in the ERL mode the beam is guided to the windowless chamber of the Mainz Gas Injection Target Experiment (MAGIX). There, the beam interacts with a thin gas jet reaching luminosities in the order of $10^{35} / \mathrm{cm}^{2} \mathrm{~s}$. The scattered electrons can be detected with a GEM based Time Projection Chamber (TPC) in the focal plane of the magnetic spectrometers. Most of the beam electrons pass through the target undisturbed and their energy will be returned stepwise to the cavities. MAGIX will cover a variety of precision experiments including the search for visible and invisible decays of dark photons.

After three recirculations in the EB mode the beam will reach the $60 \mathrm{~cm}$ long liquid hydrogen target of the P2 experiment. In about 20,000 hours the weak mixing angle in electron-proton scattering will be measured with unprecedented precision. Energy recovery will not be possible in this case and a high-power beam dump at the end of the P2 beam line is required. This aluminium beam dump is ideally suited as a target for a DM experiment - DarkMESA. 


\section{DarkMESA Concept}

Light Dark Matter (LDM) could be interacting with the Standard Model (SM) through a (massive) dark photon $\gamma^{\prime}$ coupling with strength $\varepsilon$. In the simplest model $\gamma^{\prime}$ is produced radiatively by Dark Bremsstrahlung in the beam dump. Under the condition $\mathrm{m}_{\gamma^{\prime}}>2 \mathrm{~m}_{\chi}$, the invisible decay $\gamma^{\prime} \rightarrow \chi \bar{\chi}$ is possible [4]. Due to the low interaction probability with SM particles, the LDM particles can reach the well shielded DarkMESA experimental site, while almost all beam-related SM particles are stopped in the concrete walls before. A fraction of the LDM particles could scatter off electrons in the high-density Cherenkov radiators of the DarkMESA calorimeter. Therefore the detector response of $\mathrm{PbF}_{2}$ crystals and various types of lead glass were studied at MAMI for beam electrons below $14 \mathrm{MeV}$. The results were compared with a Geant4 simulation of energy-loss, light production, transport, and detection. With a signal height of 1.8 and 1.5 photoelectrons per $\mathrm{MeV}$ energy deposition, $\mathrm{PbF}_{2}$ and the lead glass Schott SF5 were found to perform best [5]. In a prototype stage (Phase A) $25 \mathrm{PbF}_{2}$ crystals and in Phase $\mathrm{B} 900 \mathrm{PbF}_{2}$ crystals and 1024 SF5 blocks with a total active volume of $0.7 \mathrm{~m}^{3}$ are planned. The crystals and photomultipliers will be re-used from former experiments and will be arranged in a modular wall layout, shown in Fig. $2 b$.

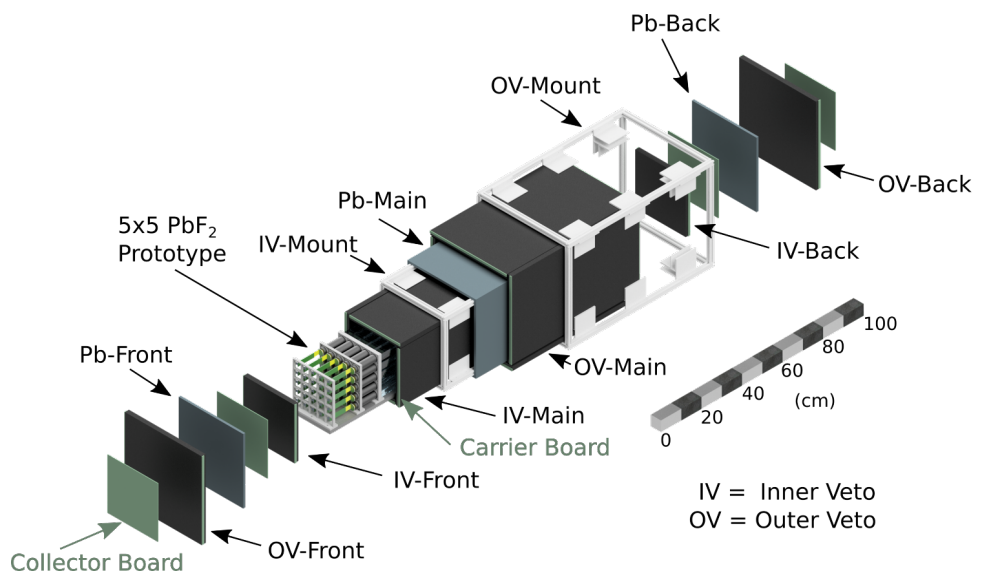

(a) DarkMESA Phase A

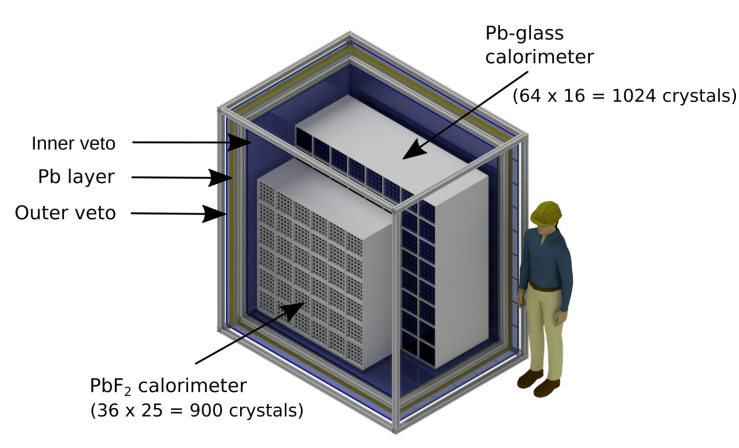

(b) DarkMESA Phase B

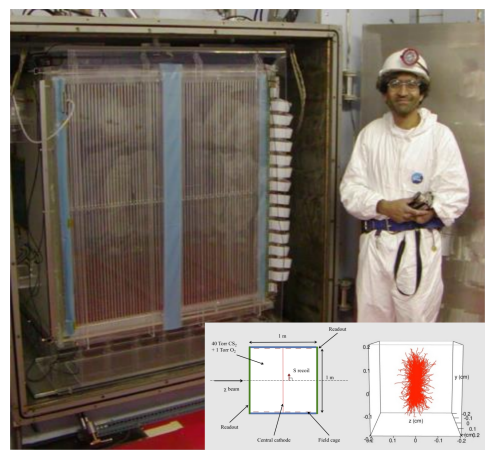

(c) DRIFT detector

Figure 2: Exploded view of the DarkMESA Phase A setup with a $\mathrm{PbF}_{2}$ prototype calorimeter inside a hermetic veto system (a), DarkMESA Phase B with 2000 crystals (b) and a photo of the DRIFT detector inside a steel vacuum vessel with directional signatures for LDM particles (taken from [6]) (c). 


\section{Radiation Studies and Veto Concept}

The LDM events are expected to be extremely rare, which is why a precise knowledge of the background is required. From the electron beam no significant background is expected - the beam energy is below the pion production threshold, no beam related neutrinos are expected and in first FLUKA simulations no beam-induced neutrons reach the DarkMESA hall. The major background is expected from cosmic-rays. The response characteristics of $\mathrm{PbF}_{2}$ and SF5 to fast neutrons $(\mathrm{MeV}$ range) was studied experimentally and in comparison to plastic scintillators a $10^{3}$ times smaller detection efficiency was found. For the veto system single panels of $2 \mathrm{~cm}$ thick EJ-200 plastic scintillators, read out by Silicon Photomultipliers (SiPMs), were studied in the MAMI electron beam [7]. A hermetic prototype veto system composed of two scintillator layers and a $1 \mathrm{~cm}$ thick lead absorber layer is studied at the moment (see Fig. 2a). Cosmic-ray simulation studies showed a veto efficiency of $99.62 \%$ for Phase A. For Phase B a $5 \mathrm{~cm}$ thick lead layer is foreseen, increasing the efficiency to more than $99.9 \%$. For Phase B in the scheduled beam time of $6,600 \mathrm{~h}$ still $10^{5}$ background events remain. An additional detector on top of the hermetic veto and calorimeter system to reject cosmogenic background or the use of outer calorimeter crystals as veto could increase the efficiency. The use of beam-off data, a rotation of the calorimeter and the analysis of signal shapes will allow a better understanding of the background with a direct measurement.

\section{Expected Reach and Outlook}

Within a MadGraph and Geant4 simulation the accessible parameter space for DarkMESA was studied. The energy and angular distribution of $\mathrm{e}^{+}$and $\mathrm{e}^{-}$particles produced in the beam dump was used to calculate the final state four-vectors of the dark photon decay with MadGraph. The four-vectors are used as input for a Geant $4 \mathrm{DM}$ generator, placed at the first radiation length of the beam dump. The cross section for interacting with the DarkMESA calorimeter was calculated and the total number of detected DM particles was obtained. Fig. 3 shows the parameter space for the invisible decay $\gamma^{\prime} \rightarrow \chi \bar{\chi}$ with the DM mass $\mathrm{m}_{\chi}$ on the horizontal axis and variable $\mathrm{y}$, proportional to the kinetic mixing $\varepsilon^{2}$, the coupling of $\gamma^{\prime}$ to $\chi$ particles $\left(\alpha_{\mathrm{D}}\right)$ and the mass ratio $\left(\mathrm{m}_{\gamma^{\prime}} / \mathrm{m}_{\chi}\right)^{4}$, on the vertical axis.

\begin{tabular}{cccrc}
\hline stage & description & period & scheduled time & EOT \\
\hline \hline Phase A & prototype & 1. - 4. year & $2,200 \mathrm{~h}$ & $7.42 \cdot 10^{21}$ \\
Phase B & PbF $_{2}+$ SF5 & 4. - 6. year & $6,600 \mathrm{~h}$ & $2.22 \cdot 10^{22}$ \\
DarkMESA DRIFT & DRIFT detector & 4. - 6. year & $6,600 \mathrm{~h}$ & $2.22 \cdot 10^{22}$ \\
Phase C & Phase B + extension & 7. - 12. year & $13,200 \mathrm{~h}$ & $4.45 \cdot 10^{22}$ \\
\hline
\end{tabular}

Table 1: Timetable of the proposed stages.

Beside the DarkMESA calorimeter Phase A and B, the projection for a complementary detection method, DarkMESA DRIFT, is shown. It will use the refurbished Directional Recoil Identification From Tracks (DRIFT) Negative Ion Time Projection Chamber (NITPC) (see Fig. 2c). The active volume will be $1 \mathrm{~m}^{3} \mathrm{CS}_{2}$ at a low pressure of $53 \mathrm{mbar}$. It can detect nuclear recoils with energies down to $20 \mathrm{keV}$. Spatial and directional signatures give this type of detector powerful 
background rejection abilities at very low energies. The DRIFT detector is capable of detecting neutrons, and cosmic-ray simulations show that about $7 \mathrm{~s}^{-1} \mathrm{~m}^{-2}$ reach the experimental site. This is why a specific high-efficiency neutron veto system is under development. The experiment could run in parallel to DarkMESA Phase B in the second funding period with a scheduled time of $6,600 \mathrm{~h}$.

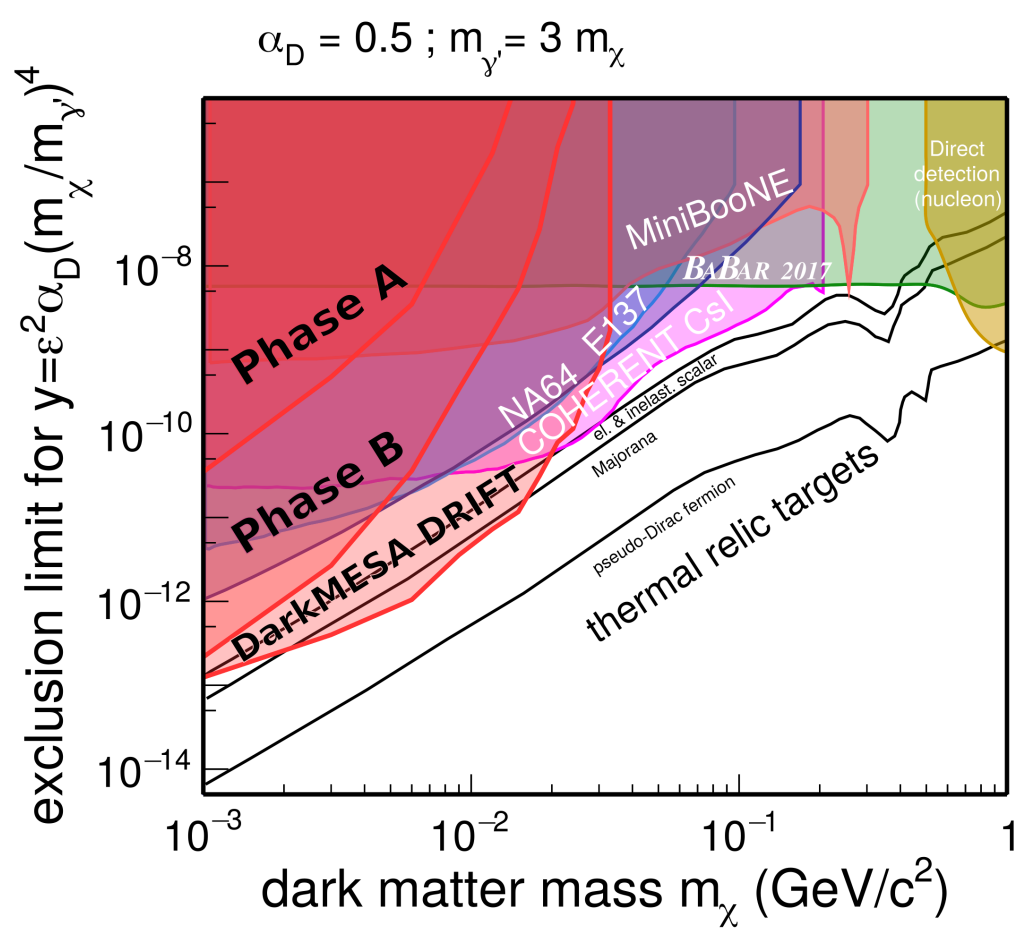

Figure 3: Parameter space for dark photons decaying invisibly to $\chi \bar{\chi}$ with the DM mass $\mathrm{m}_{\chi}$ on the horizontal axis and variable $\mathrm{y}$, proportional to the kinetic mixing $\varepsilon^{2}$, the coupling of $\gamma^{\prime}$ to $\chi$ particles $\left(\alpha_{\mathrm{D}}\right)$, and the mass ratio $\left(\mathrm{m}_{\gamma^{\prime}} / \mathrm{m}_{\chi}\right)^{4}$, on the vertical axis. The predictions for the exclusion limit with $90 \%$ C.L. are shown in red. The detection efficiency is $95 \%$, the conservatively estimated electron recoil energy threshold for DarkMESA is $10 \mathrm{MeV}$ and the nuclear recoil energy threshold for DarkMESA DRIFT is $20 \mathrm{keV}$. The EOT were set according to the time schedule in Tab. 1. The existing exclusion limits from various other experiments [8-12] are shown.

The projections in Fig. 3 assume zero background, a confidence level of $90 \%$, a detection efficiency of $95 \%, \alpha_{\mathrm{D}}=0.5$, and $\mathrm{m}_{\gamma^{\prime}}=3 \mathrm{~m}_{\chi}$. The electron recoil energy threshold for Phase A and $\mathrm{B}$ is conservatively set to $10 \mathrm{MeV}$. The black lines, the so-called targets, represent the minimum couplings that are still consistent with the observable abundance of thermal DM. DarkMESA Phase A lies within the existing limits, while Phase B can achieve stronger limits in the low mass range. With its low threshold and large active volume, DarkMESA DRIFT can expand the accessible parameter space and reach the thermal relic targets.

As seen in Tab. 1, the major part of scheduled time is available for a Phase C. A measurement with the Phase B setup for an extended period of time can improve the limits only slightly. That is why in addition an innovative concept of using thin radiation shielding glasses is studied at the moment. A variety of plates is stacked together along the beam axis, resulting in a directionality of the signals and a reduction of the volume cost ratio due to the commercial availability. 
The ability to perform experiments with different detector concepts and detection methods at one experimental site is a great advantage of the beam dump experiment at MESA.

\section{Acknowledgments}

The authors would like to thank the MAMI operators, technical staff, and the accelerator group for their excellent work. We are thankful to M. Battaglieri and A. Celentano for the discussions and help with the MadGraph simulation. This work was supported by the PRISMA ${ }^{+}$Cluster of Excellence, Germany "Precision Physics, Fundamental Interactions and Structure of Matter", and by the Helmholtz-Gemeinschaft Deutscher Forschungszentren (HGF), Germany with a HGFExzellenznetzwerk.

\section{References}

[1] F. Hug et al., MESA - an ERL project for particle physics experiments, LINAC16 Conference Proceedings MOP106012, pp. 313-315 (2017).

[2] B. S. Schlimme et al. (A1 and MAGIX Collab.), Operation and characterization of a windowless gas jet target in high-intensity electron beams, Nucl. Instrum. Meth. Phys. Res. A 1013, 165668 (Oct. 2021).

[3] D. Becker et al., The P2 experiment, Eur. Phys. J. A 54, 208 (Nov. 2018).

[4] L. Doria et al., Dark Matter at the Intensity Frontier: The new MESA electron accelerator facility, PoS 360 (ALPS 2019) 022.

[5] M. Christmann et al., Detector response of Cherenkov radiators for calorimetry in the energy range below $14 \mathrm{MeV}$, Nucl. Instrum. Meth. Phys. Res. A 960, 163665 (Apr. 2020).

[6] D. Snowden-Ifft, BDX-DRIFT: A low-energy, low-background, directional search for LDMA, UCLA Dark Matter 2018, Los Angeles, CA, USA (Feb. 2018).

[7] M. Lauß et al. (MAGIX Collab.), Electron beam studies of light collection in a scintillating counter with embedded fibers, Nucl. Instrum. Meth. Phys. Res. A 1012, 165617 (Oct. 2021).

[8] S. N. Gninenko et al., Search for Dark Sector Physics with NA64, Phys. Part. Nuclei 51, 829-858 (Sep. 2020).

[9] J. P. Lees et al. (BaBar Collab.), Search for Invisible Decays of a Dark Photon Produced in $e^{+} e^{-}$Collisions at BaBar, Phys. Rev. Lett. 119, 131804 (Sep. 2017).

[10] B. Batell et al., Strong Constraints on Sub-GeV Dark Matter from SLAC Beam Dump E137, Phys. Rev. Lett. 113, 171802 (Oct. 2014).

[11] A. A. Aguilar-Arevalo et al. (MiniBooNE-DM Collab.), Dark Matter Search in a Proton Beam Dump with MiniBooNE, Phys. Rev. Lett. 118, 221803 (May 2017).

[12] D. Akimov et al., First Probe of Sub-GeV Dark Matter Beyond the Cosmological Expectation with the COHERENT CSI Detector at the SNS, arXiv:2110.11453 (Oct. 2021). 